\title{
EVALUATION OF THE ANTIOXIDANT ACTIVITY OF FICUS RACEMOSA PLANT EXTRACTS FROM NORTH-WESTERN DISTRICT OF BANGLADESH
}

\author{
Johora Sultana ${ }^{1}$, A.S. Kabir ${ }^{1}$ Md. Abdul Hakim ${ }^{1}$, M. Abdullah ${ }^{2}$, N Islam ${ }^{2}$ and Md. Abu Reza ${ }^{*}$ \\ ${ }^{1}$ Department of Genetic Engineering and Biotechnology, University of Rajshahi, Rajshahi-6205, Bangladesh \\ ${ }^{2}$ Department of Zoology, University of Rajshahi, Rajshahi-6205, Bangladesh \\ ${ }^{*}$ Corresponding Author: e-mail: reza.gen@ru.ac.bd
}

\begin{abstract}
Antioxidant is a new hype in naturopathy and at present it has huge demand in cosmetic, nutritional supplementation and pharmacological industry. It reduces cell damages caused by free-radical which are responsible for various ailment like ageing, cancer, coronary heart disease, diabetes mellitus, neurodegenerative disorders, atherosclerosis, cataracts and inflammation. At present any comprehensive data on the antioxidant content and extent of their activity of the indigenous medicinal plants of Bangladesh is not available. Therefore, here antioxidant activity of a popular medicinal plant - Ficus racemosa was determined. Methanolic extract was prepared from the leaf and stem bark of $F$. racemosa. Their total phenolics and free radical scavenging capacity was determined using folin-ciocalteu reagent (FCR) assay and DPPD-radical scavenging assay respectively. Stem bark and leaves of F. racemosa contains 242.97 and $235.45 \mathrm{mg}$ of GAE / gm of dried extract of phenolics respectively. Current studies also show that $F$. racemosa barks contain a high antioxidant activity. IC $_{50}$ value of bark extract was found to be $19 \mu \mathrm{g} / \mathrm{ml}$ which is even better that of the standard BHT. Therefore, present studies suggest that F. racemosa bark has a huge potential in terms of commercial use as source of antioxidant.
\end{abstract}

Key word: Ficus racemosa, plant extract, phenolic conttent, antioxidant.

সারাংশ: প্রকৃতিতত্বে (নেচারোপ্যাথীতে) অ্যান্টিঅক্সিডেন্ট একটি সাম্প্রতিক আকর্ষণ এবং বর্তমানে প্রসাধন, পুষ্টি সহযোগী এবং ঔষধশিল্পে এর প্রচুর চাহিদা রয়েছে। এরা দেহের অভ্যন্তরে প্রতিনিয়ত সৃষ্ট মুক্ত অণু কর্তৃক সৃষ্ট কোষীয় ক্ষতি (ড্যামেজ) হ্রাস করে, যা বিভিন্ন শারিরিক অসুস্থতা যেমন বয়সবৃদ্ধি, ক্যান্সার, হৃদরোগ, ডায়াবেটিস, স্নাযুরোগ, হাড় ক্ষয়, ছানি ও প্রদাহের জন্য দায়ী। এযাবৎ পর্যন্ত বাংলাদেশী ঔষধি উদ্ভিদসমূহে বিদ্যমান অ্যান্টিঅক্সিডেন্টের প্রকৃতি এবং তার কার্যকারিতা সম্পর্কে পরিপূর্ণ কোন ধারনা নেই। তাই এখানে একটি জনপ্রিয় ঔষধী উদ্ভিদ ডুমুর (ফাইকাস রেসিমোসা) এর অ্যান্টিঅক্সিডেন্টের কার্যকারীতা যাচাই করা হয়েছে। এই কাজের জন্য ডুমুর গাছের পাতা ও বাকল এর মিথানল নির্যাস তৈরি করা হয়েছে এবং তার মধ্যে বিদ্যমান সম্পূর্ণ ফেনল উপাদানের পরিমাণ ও তার মুক্ত অণুর অপসারণ দক্ষতা পরিমাপ করা হয়েছে যথাক্রনে ফলিন-ক্যালচু পরিক্ষণ ও ডিপিপিএইচ- মুক্ত অণুর অপসারণ দক্ষতা পরিক্ষণ এর মাধ্যমে। ডুমুর গাছের বাকল ও পাতার প্রতি গ্রাম শুক্ষ নির্যালে যথাক্রমে ২৪২.৯৭ এবং ২৩৫.৪৫ মি.গ্রা. সমপরিমাণ জিএই ফেনলিক উপাদান রয়েছে। পরীক্ষণে দেখা যাচ্ছে যে, ডুমুর গাছের বাকলের উচ্চ অ্যান্টিঅক্সিডেন্ট কার্যকারিতা রয়েছে। এর বাকলের IC ৫০ মান ১৯ মাইক্রোগ্রাম/মিলি লিটার যা এমনকি এই পরীক্ষণে ব্যবহৃত স্ট্যান্ডার্ড বিএইচটি এর চেয়ে ভালো। সুতরাং, এই পরীক্ষণ এটা প্রমাণ করে যে, অ্যান্টিঅক্সিডেন্টের উৎস হিসাবে বাণিজ্যিক ব্যবহারের ক্ষেত্রে ডুমুর গাছের বাকলের প্রচুর সম্ভাবনা রয়েছে।

\section{Introduction}

Majority of the diseases/disorders are mainly linked to oxidative stress caused by free radicals (Gutteridgde et al. 1995). Free radicals are produced continuously in our body as a byproduct of our daily metabolic activity and cellular oxidation reactions. Apart from this, different factors such as alcohol, tobacco, prescription drugs, pesticides, harmful chemicals, additives in the foods and pollutants in the air also contribute production of excessive amount of free radicals in our body (Tiwari et al. 2001). In treatment of these diseases caused by oxidative stress, antioxidant therapy has gained an immense importance. Antioxidants have been reported to prevent oxidative damage caused by free radical and may prevent the occurrence of diseases like ageing, cancer, coronary heart disease, diabetes mellitus, neurodegenerative disorders, cardio-vascular diseases, atherosclerosis, and inflammation (Buyukokuroglu et al. 2001). It can interfere with the oxidation process by reacting with free radicals, chelating metals and also by acting as oxygen scavengers (Buyukokuroglu et al. 2001; Shahidi et al. 1992).
Natural antioxidants are mainly found in plants as polyphenols. Polyphenols especially, flavonoids and other phenolic compounds are widely distributed in plants which have been reported to exert multiple biological effect, including antioxidant, free radical scavenging abilities, anti-inflammatory, anticarcinogenic etc (Willett et al.2002; Miller et al.1996, Farjana 2003). However, the nature, quality and quantity of polyphenol content may vary significantly even in the same plant species due to different geographic distribution. This means, the same plant species from one part of the world may have different polyphenol constituents and thus may have different antioxident properties. This is probably due the difference in the environmental factors that they are interacting with.

Research with important bioactive compounds in plant and food materials has received much more attention. Currently, research on antioxidant activity is an important topic in the medical sector as well as in the food industry due to the fact that, antioxidants play a major part in inhibiting and scavenging free radicals, 
thus providing protection to human against infections and degenerative diseases. In addition it is a proved fact now that antioxidants are the key for fighting ageing. Recently research is now directed towards natural antioxidants originated from plants due to safe therapeutics. Therefore, antioxidants with free radical scavenging activities may have great relevance in the prevention and therapeutics of diseases in which oxidants or free radicals are implicated (Soares et al. 1997). In the current market several synthetic antioxidant are present. These synthetic anti-oxidants, such as propyl gallate, butylated hydroxyanisole (BHA), butylated hydroxytoluene (BHT) and tertiary butylhydroquinone has been widely used industrially to control lipid oxidation in foods. However, the use of these synthetic antioxidants has been questioned due to their potential health risks and toxicity (Kahl and Kappus 1993). The search for antioxidants from natural source has received much attention and efforts have been put into identify compounds that can act as suitable antioxidants to replace synthetic ones. At present time we do not have enough data on the antioxidant content and extent of their activity of the indigenous medicinal plant of Bangladesh, although antioxidants have a huge potential in industrial and pharmacological application. Ageing, cancer, coronary heart disease, diabetes mellitus, neurodegenerative disorders, cardio-vascular diseases, atherosclerosis, and inflammation are closely related with the deficiency of antioxidant.

Ficus constituted one of the largest genera of medicinal plants primarily prevailing in subtropical and tropical regions throughout the world and used for a vast range of various ailments. Different parts of the plant like bark, leaves, tender shoots, fruits, seeds, and latex are known to have medicinal importance. A number of works have already been carried out to evaluate the antioxidant property of $F$. racemosa. However, comprehensive studies on $F$. racemosa from northern part of Bangladesh are yet to be done. Therefore, here we have evaluated the potency of the antioxidant content from the methanolic extract of leaf and bark sample of $F$. racemosa from Rajshahi district of Bangladesh. Rajshahi district is situated at the northwestern part of Bangladesh and has an extreme semiarid climate. Current study will help us to understand and compare the variation in antioxidant content and property of this plant from other parts of the world which have a different climatic condition.

\section{Materials and Methods}

\section{Chemicals}

2, 2-Diphenyl-2-picryl hydrazyl (DPPH), Folinciocalteu reagent (FCR) was obtained from SigmaAldrich (St. Louis, USA.) Butylated hydroxy toluene (BHT), methanol was purchased from Merck (Germany) and gallic acid was procured from Wako pure chemicals Ltd., Japan. All other chemicals used in the current paper were of analytical grade.

\section{Collection of samples and preparation of extracts}

Leaves (FL) and stem barks (FB) of Ficus racemosa plant were collected from different places of Rajshahi in May, 2011. Upon arrival at the laboratory, samples were washed thoroughly under running tap water and then dried at $45^{\circ} \mathrm{C}$ for $48 \mathrm{~h}$ (Mohd Zin et al. 2002). The dried samples were stored in sealed polyethylene bags with silica gel. Dried leaf and bark samples were ground into coarse powder using a grinding machine. $100 \mathrm{gm}$ of powdered plant materials (Leaves and stem barks) were taken in $1 \mathrm{~L}$ conical flasks and allowed for soaking in $500 \mathrm{ml}$ of methanol for each sample. The conical flasks with its contents were then sealed and kept on orbital shaker for continuous shaking at 150 rpm for 2 days. Sonication was also performed with the help of an Ultrasound Bath for breaking the cell walls completely. Followed by sonication the conical flasks were kept again on orbital shaker for 1 day. The mixtures were then filtered through Whatman No.1 filter paper and solvents were completely evaporated using a rotary evaporator under controlled temperature $\left(45^{\circ} \mathrm{C}\right)$.

\section{Antioxidant activity test \\ Total phenolic content determination}

Total phenolic content of methanolic extracts of different parts, including FL ( $F$. racemosa leaf), FB ( $F$. racemosa bark) were determined by the method reported by Chang et al. 2002; involving folin-ciocalteu reagent (FCR) as oxidizing agent and gallic (GA) acid as standard. The results were expressed as $\mathrm{mg}$ of GAE/gm of dried extractives.

\section{DPPH (1, 1-diphenyl-2-picrylhydrazyl) free radical scavenging assay}

The free radical scavenging capacity of the extracts was determined using DPPH (Choi et al, 2000; Desmarchelier et al. 1997). DPPH solution $(0.004 \%$ $\mathrm{w} / \mathrm{v})$ was prepared in $95 \%$ methanol. $1.0 \mathrm{ml}$ of methanol solution of different extracts and standard at different concentrations $(25 \mu \mathrm{g} / \mathrm{ml}, 50 \mu \mathrm{g} / \mathrm{ml}, 100$ 
$\mu \mathrm{g} / \mathrm{ml}, 150 \mu \mathrm{g} / \mathrm{ml}$ and $200 \mu \mathrm{g} / \mathrm{ml}$ ) were taken in test tubes. $1.5 \mathrm{ml}$ of methanol solution of DPPH was added into each of the test tubes. The test tubes were then incubated at RT (room temperature) for 30 minutes in dark place to complete the reaction. Then the absorbance of the solutions were measured at $519 \mathrm{~nm}$ using a spectrophotometer against blank. BHT was used as a reference standard and dissolve in distilled water to make the stock solution with the same concentration. A typical blank solution contained all reagents except plant extract or standard solution also taken as control.

The percentage (\%) inhibition activity was calculated from the following equation

$$
\% I=\left\{\left(A_{0}-A_{1}\right) / A_{0}\right\} X 100
$$

Where,

$A_{0}$ is the absorbance of the control, and

$A_{1}$ is the absorbance of the extract/standard.

Then \% inhibitions were plotted against concentration, and $\mathrm{IC}_{50}$ was calculated from the graph (Viturro et al. 1999).

\section{Evaluation of Repellent Activity of $F$. racemosa extracts}

To check whether $F$. racemosa has any insectidal activity, repellent activity test against the stored grain pest Tribolium castaneum was carried out. The repellency test used was adopted from the method of McDonald et al. (1970) with some modifications by Talukder \& Howse (1994). Half filter paper discs (Whatman No. 40, diameter $90 \mathrm{~cm}$ ) were prepared and selected doses of all the methanol extract separately applied onto each of the half-disc and allowed to dry out by exposed in the air for 10 minutes. Each treated half-disc was then attached lengthwise, edge-to-edge, to a control half-disc with adhesive tape and placed in a Petri dish (diameter $90 \mathrm{~cm}$ ). Ten adult insects were released in the middle of each filter-paper circle. Each concentration was tested five times. Insects that settled on each half of the filter paper disc were counted after $1 \mathrm{~h}$ and then at hourly intervals for $5 \mathrm{~h}$. The average of the counts was converted to percentage repellency $(P R)$ using the formula (Talukder and Howse 1995):

$P R=2(\mathrm{C}-50)$,

Where, $\mathrm{C}$ is the percentage of insects on the untreated half of the disc. Positive values expressed repellency and negative values for attractant activity. The values in the recorded data were then calculated for percent repellency.

\section{Evaluation of In vitro Antibacterial Activity of $F$.} racemosa extract

Five (5) $\mathrm{mg}$ of each extracts of $F$. racemosa were added carefully to $5 \mathrm{ml}$ of de-ionized water and dissolved by vortexing the tube. The stock extract solution was prepared as $1 \mu \mathrm{g} / \mu \mathrm{l}$. The stock of Bacillus subtilis and Enterobacter sp. strains were taken out of $-20^{\circ}$ and revived in LB medium. Culture was incubated at $37^{\circ} \mathrm{C}$ for overnight. These suspensions were used as inoculum. Disk diffusion method was used in the current experiment to evaluate the antibacterial activity of FL, FB extracts. The discs (6mm diameters) were made by punching the Whatman No. 1 filter paper with the help of punch machine. These discs were taken into the screw capped tube and sterilized in an autoclave machine at $121^{\circ} \mathrm{C}$ for 20 minutes to ensure sterilization. The paper discs were soaked with different concentrations $(50 \mu \mathrm{g}, 100 \mu \mathrm{g}, 150 \mu \mathrm{g}$ and $200 \mu \mathrm{g})$ of each extract with the help of micropipette and keep them at laminar air flow hood for dryness (5-10 minutes). For standard the disc papers were prepared in a similar way using $30 \mu$ g of Chloramphenical. The disks containing sample as well as the standard were introduced on the upper layer of the seeded agar plate by sterile forceps. After that the plates were incubated overnight at $37^{\circ} \mathrm{C}$ and diameter of zone of inhibition (cleared area around the disk) was measured.

\section{Results}

\section{Determination of Total Phenolics}

The total phenolic content of methanolic extracts of different parts of $F$. racemosa was determined according to the method reported by Singleton et al.1999 using folin-ciocalteu reagent (FCR) as oxidizing agent and gallic (GA) acid as standard. Absorbance was measured at $750 \mathrm{~nm}$ wavelength after 20 minute of incubation at room temperature. Both leaves and stem bark methanolic extracts showed similar result in terms of total phenolic content. Among the two samples the highest phenolic content was found in F. racemosa stem bark which were $242.97 \mathrm{mg}$ of GAE / gm of dried extract followed by leaves which were $235.45 \mathrm{mg}$ of GAE / gm of dried extract at concentration of $200 \mu \mathrm{g} / \mathrm{ml}$ (Fig.1). So, it is obvious that stem bark of $F$. racemosa possessed slightly higher phenolic content than that of leaves. Result of the total phenolic content determination are tabulated in table 1 and depicted in figure 1. 
Table 1 Determination of Total Phenolics. Absorbance of GA (standard) at different concentrations and absorbance of FL and FB extracts at $200 \mu \mathrm{g} / \mathrm{ml}$ after treatment with Folin-Ciocalteu reagent. Absorbance of each sample was taken in triplicated indicated as a, b and c.

\begin{tabular}{|c|c|c|c|c|c|c|}
\hline \multirow[b]{2}{*}{ Samples } & \multirow[b]{2}{*}{$\begin{array}{l}\text { Conc. } \\
(\mu g / m l)\end{array}$} & \multicolumn{3}{|c|}{ Absorbance } & \multirow[b]{2}{*}{$\begin{array}{c}\text { Absorbance Mean } \pm \\
\text { STD }\end{array}$} & \multirow{2}{*}{$\begin{array}{c}\text { GAE/gm of dried } \\
\text { extract }\end{array}$} \\
\hline & & $\mathbf{A}$ & b & $\mathbf{C}$ & & \\
\hline \multirow{6}{*}{ Gallic Acid } & 10 & 0.106 & 0.112 & 0.121 & $0.113 \pm 0.006$ & \\
\hline & 20 & 0.303 & 0.309 & 0.31 & $0.307 \pm 0.003$ & \\
\hline & 40 & 0.538 & 0.541 & 0.544 & $0.541 \pm 0.002$ & \\
\hline & 80 & 1.101 & 1.013 & 1.012 & $1.042 \pm 0.041$ & \\
\hline & 160 & 1.987 & 2.012 & 1.995 & $1.998 \pm 0.010$ & \\
\hline & 320 & 3.845 & 3.621 & 3.813 & $3.759 \pm 0.098$ & \\
\hline FL & 200 & 0.785 & 0.886 & 0.987 & $0.886 \pm 0.101$ & 235.45 \\
\hline FB & 200 & 1.162 & 1.194 & 1.226 & $1.194 \pm 0.032$ & 242.97 \\
\hline
\end{tabular}
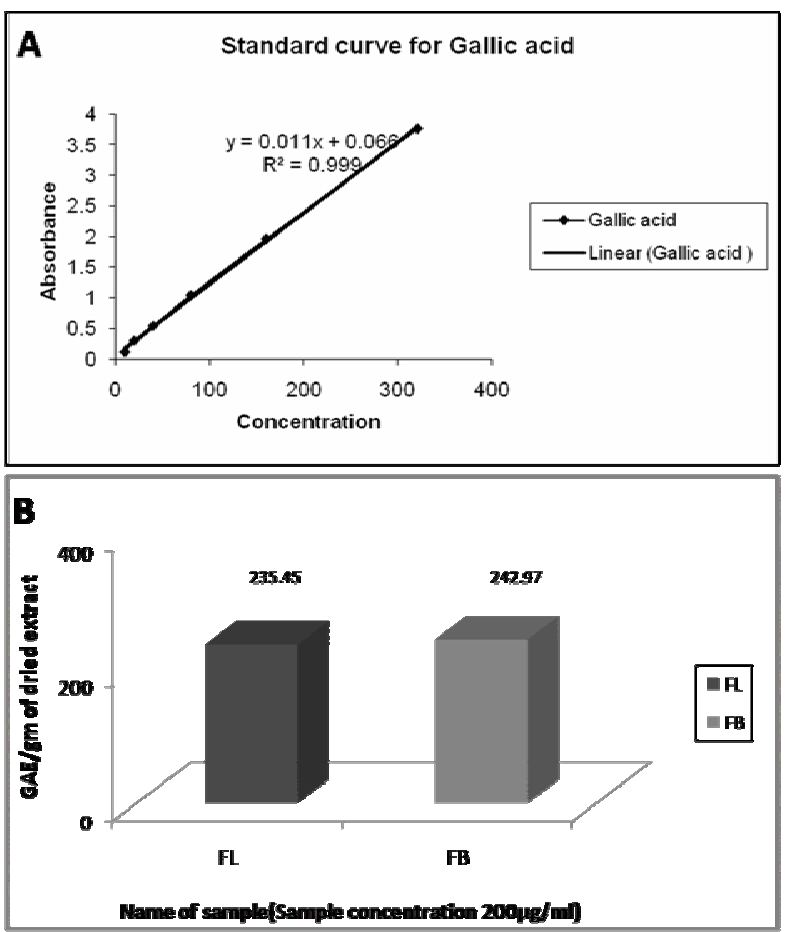

Fig. 1 Determination of total phenolics of F. racemosa leaf and bark methanolic extracts. A) standard carve of galic acid and B) bar diagram showing the comparison between FL and FB samples

\section{DPPH (1, 1-diphenyl-2-picrylhydrazyl) Radical Scavenging Assay}

The 1, 1-diphenyl-2-picrylhydrazyl radical (DPPH) has been widely used to evaluate the free radical scavenging capacity of antioxidants. DPPH free radical is reduced to the corresponding hydrazine when it reacts with hydrogen donors. DPPH can make stable free radicals in aqueous or methanol solution. With this method, it is possible to determine the antiradical power

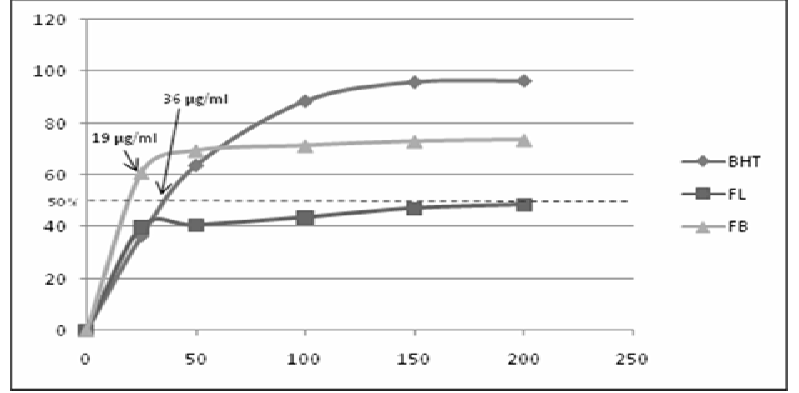

Fig. $2 \mathrm{IC}_{50}(\mu \mathrm{g} / \mathrm{ml})$ values of methanolic extractives of $\mathrm{FB}$, along with BHT as standard for DPPH radical scavenging activity. $\mathrm{IC}_{50}$ value is shown using red dotted line and indicated with arrow.
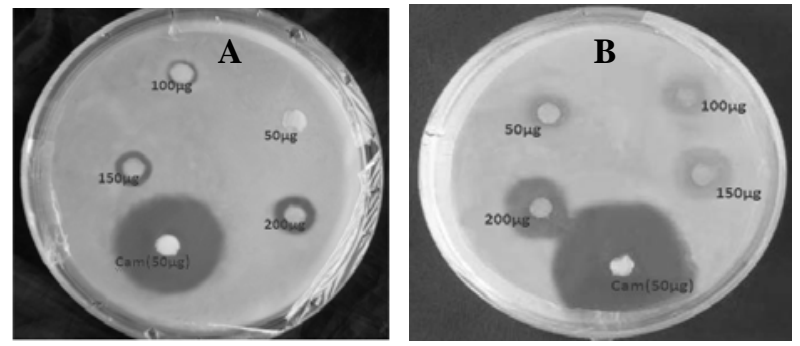

Fig. 3 Photograph showing the antibacterial activity of Ficus racemosa stem bark (FB) extract against the strain of (A) Bacillas subtilis and (B) Enterobacter sp. respectively.

of an antioxidant activity by measurement of the decrease in the absorbance of DPPH at $519 \mathrm{~nm}$. Resulting from a color change from purple to yellow, the absorbance decreased when the DPPH was scavenged by an antioxidant, through donation of hydrogen to form a stable DPPH molecule. In the radical form, this molecule had an absorbance at 519 $\mathrm{nm}$ which disappeared after acceptance of an electron or hydrogen radical from an antioxidant compound to become a stable diamagnetic molecule. $1.0 \mathrm{ml}$ of 
methanolic extract solution of $F$. racemosa leaf and bark sample was taken for the experiment. Decolorization of DPPH by the effect of the extracts were measured at $517 \mathrm{~nm}$. The results of DPPH radical scavenging assays of butylated hydroxytoluene (BHT) (standard) and plant extracts are tabulated in table 2. Figure 2 showed free radical scavenging activity of the two extractives used in the current paper. At a concentration of $200 \mu \mathrm{g} / \mathrm{ml}$,the scavenging activity of the F. racemosa leaves and bark extracts were found to be $48.54701 \%$ and $73.46154 \%$ respectively, while at the same concentration, the activity of BHT was $96.35 \%$. Thus, F. racemosa stem bark exhibited significant free radical scavenging activity (Figure 2A). The $\mathrm{IC}_{50}$ of stem bark of $F$. racemosa was $19 \mu \mathrm{g} / \mathrm{ml}$.

Table 2 DPPH free radical scavenging activity of methanolic extracts of standard BHT and FL and FB at various concentrations. Absorbance of each sample was taken in triplicated indicated as a, b and c.

\begin{tabular}{|c|c|c|c|c|c|c|}
\hline \multirow{2}{*}{$\begin{array}{l}\text { Name of } \\
\text { Samples }\end{array}$} & \multirow{2}{*}{$\begin{array}{l}\text { Conc. } \\
\mu g / m l\end{array}$} & \multicolumn{3}{|c|}{ \% of Scavenging } & \multirow{2}{*}{$\begin{array}{c}\text { Mean \% of scavenging } \pm \\
\text { STD } \\
\end{array}$} & \multirow{2}{*}{$\begin{array}{c}\mathrm{IC}_{50} \\
(\mu \mathrm{g} / \mathrm{ml})\end{array}$} \\
\hline & & $\mathbf{a}$ & b & $\mathrm{C}$ & & \\
\hline \multirow{5}{*}{ BHT } & 25 & 36.46 & 36.42 & 36.47 & $36.45 \pm 0.026$ & \multirow{5}{*}{36} \\
\hline & 50 & 63.69 & 63.72 & 63.63 & $63.68 \pm 0.045$ & \\
\hline & 100 & 88.51 & 88.47 & 88.53 & $88.50 \pm 0.030$ & \\
\hline & 150 & 95.81 & 95.84 & 95.83 & $95.83 \pm 0.015$ & \\
\hline & 200 & 96.35 & 96.32 & 96.37 & $96.35 \pm 0.025$ & \\
\hline \multirow{5}{*}{ FL } & 25 & 40.41 & 39.98 & 39.48 & $39.96 \pm 0.465$ & \multirow{5}{*}{ - } \\
\hline & 50 & 41.32 & 41.05 & 39.42 & $40.60 \pm 1.028$ & \\
\hline & 100 & 44.11 & 43.58 & 43.21 & $43.63 \pm 0.452$ & \\
\hline & 150 & 49.23 & 47.51 & 44.79 & $47.18 \pm 2.239$ & \\
\hline & 200 & 50.42 & 49.73 & 45.49 & $48.55 \pm 2.670$ & \\
\hline \multirow{5}{*}{ FB } & 25 & 62.83 & 60.61 & 59.12 & $60.85 \pm 1.867$ & \multirow{5}{*}{19} \\
\hline & 50 & 72.23 & 68.59 & 67.13 & $69.32 \pm 2.627$ & \\
\hline & 100 & 75.36 & 71.23 & 67.12 & $71.24 \pm 4.120$ & \\
\hline & 150 & 74.34 & 72.91 & 71.47 & $72.91 \pm 1.435$ & \\
\hline & 200 & 74.94 & 73.42 & 72.03 & $73.46 \pm 1.455$ & \\
\hline
\end{tabular}

Table 3 Antimicrobial activity of FL and FB at various concentrations.

\begin{tabular}{|c|c|c|c|c|}
\hline \multirow[t]{2}{*}{ Sample } & \multirow[t]{2}{*}{$\begin{array}{l}\text { Sample concentration/dose } \\
(\mu \mathrm{g})\end{array}$} & \multicolumn{2}{|c|}{$\begin{array}{c}\text { Inhibition zone after } 24 \text { hour } \\
(\mathrm{mm})\end{array}$} & \multirow{2}{*}{$\begin{array}{c}\text { Inhibition zone by } \\
\text { Chloramphenicol at } 30 \mu \mathrm{gg} \\
\text { conc. (mm) }\end{array}$} \\
\hline & & Bacillus subtilis & Enterobacter sp. & \\
\hline FB & $\begin{array}{c}50 \\
100 \\
150 \\
200\end{array}$ & $\begin{array}{c}4 \\
8.0 \\
13 \\
15\end{array}$ & $\begin{array}{c}- \\
1.5 \\
3 \\
4\end{array}$ & ( \\
\hline FL & $\begin{array}{c}50 \\
100 \\
150 \\
200 \\
\end{array}$ & $\begin{array}{l}- \\
- \\
- \\
-\end{array}$ & $\begin{array}{l}- \\
- \\
- \\
-\end{array}$ & 0 \\
\hline
\end{tabular}

\section{Repellant activity}

Both the leaf and stem bark extracts of $F$. racemosa were tested against $T$. castaneum adults for their repellent activity. However, either of the extracts did not show any significant repellency against the adult beetles.

\section{Estimation of In vitro Antibacterial Activity of $F$. racemosa}

Antimicrobial activity test of FL and FB was done against two bacterial species: Bacillus subtilis and Enterobacter sp. in different concentrations. As control, $30 \mathrm{mg} / \mathrm{ml}$ chloramphenicol was used. However, not much activity was found in any of the FL and FB samples compared to control (table 3 and figure 3 ). Only high concentration of FB extract showed moderate antimicrobial activity (15mm clearing zone) against Enterobacter sp. FL did not show any antimicrobial activity.

\section{Discussion}

Antioxidants works as natural healer in repairing oxidative cell damages and thus prevents a lot of physical ailment (Aruoma 1987). A great number of aromatic, medicinal, spices, vegetable, fruits and other plants contain chemical compounds exhibiting antioxidant properties (Cotelle et al. 1996). Various workers also reported high antioxidant activities of different plant extracts using different assaying methods. Chu et al. (2000) reported strong DPPH free radical scavenging activity and ferric ions reducing power in sweet potato leaves. Betel leaves had been reported to be more potent than BHT (Lean and Mohamed 1999) which is similar to our result. Peng et al. (2003) identified 10 flavonoid compounds and Yagi 
et al. (1994) found three hydrophilic sulfatedflavonoids in laksa leaves which exhibited potent antioxidant properties. Betalains responsible for the intense red color of red amaranthus were also powerful scavengers of the DPPH free radical and some of them were found to be stronger than rutin, catechin and ascorbic acid (Cai et al. 2003). Miean and Mohamed (2001) reported high total flavonoid content in spring onion leaves.

Bangladesh is rich in flora and fauna with its own unique climatic conditions. However, still such rich resources of our country need to be extensively worked on. At present no clear data is available on the antioxidant content and extent of their activity of the indigenous medicinal plant of Bangladesh, although antioxidants have a huge potential in industrial and pharmacological application. Ficus is a well known medicinal plant of this subcontinent with many profound medicinal values. Therefore, the current project was taken to evaluate the potency of antioxidant from this indigenous plant of north-western part of Bangladesh. Results of the current studies show that leave and stem bark of $F$. racemosa contains high level of antioxident activity. Although, a number of works have been carried out on antioxidant properties from different plant part (especially fruits) of $F$. racemosa from Bangladesh (Jahan et al. 2009; Hamid et al. 2011; Sirisha et al. 2010) but data from the methanolic leaf and bark extract is scantly. Results indicat that both leaf and bark extract of $F$. racemosa contain nearly equivalent amount of polyphenol content (235.45 and $242.97 \mathrm{GAE} / \mathrm{gm}$ of dried extract respectively). However, bark extract shows significantly higher free radical scavenging activity compared to the leaf extract. $\mathrm{IC}_{50}$ value of bark extract was found to be $19 \mu \mathrm{g} / \mathrm{ml}$. However, the leaves did not show $\mathrm{IC}_{50}$ from the concentration of $25-200 \mu \mathrm{g} / \mathrm{ml}$. This indicates that Ficus leave extractives may require more than $200 \mu \mathrm{g} / \mathrm{ml}$ extract for showing $\mathrm{IC}_{50}$. So, it is clear that $F$. racemosa bark sample had higher scavenging activity even that of the standard BHT which is a significant in terms of commercial use of $F$. racemosa bark as source of antioxidant.

\section{Conclusion}

Currently, any antioxidant containing drugs and cosmetics is not manufactured in our country although we have affluent and diversified flora resources with very rich history of their medicinal use in 'Aiurveda'. Antioxidant can be used to produce medicines and cosmetics so that, the drug and cosmetic industries in our county can have their own herb based formulation. The measurement of the antioxidant capacity of selected plants is important to quantify the intake of natural antioxidants in a dietary supplement. Additionally, these studies will help in the selection of natural source of antioxidant and in the use of health relevant food industry. Its constituents scavenge free radicals and thus may contribute a protective effect against oxidative damage induced to cellular macromolecules. However, further studies for the isolation and identification of individual bioactive compounds and also in vivo studies are needed for understanding their mechanism of actions well. It is expected that, the current study will provide baseline information for the local pharmaceutical companies of Bangladesh to evaluate the possibilities of using $F$. racemosa stem bark in preparation of dietary supplements and cosmetic products.

\section{References}

1. Aruoma OI 1987. Free radicals, oxidative trace and antioxidants in human health and diseases. J. Am. Oil Chemist's Soc. 75: 199-212.

2. Buyukokuroglu ME, Gulcin I, Oktay M and Kufrevioglu OI. 2001. In vitro antioxidant properties of dantrolene sodium. Pharmacol. Res. 44: 491- 95.

3. Cai Y, Sun M and Corke H 2003. Antioxidant activity of betal vines from plants of the Amaranthaceae. J. Agri. Food Chem. 51: 2288-2294.

4. Chang C, Yang M, Wen H and Chern J 2002. Estimation of total flavonoid content in Propolis by two complementary colorimetric methods. J. Food Drug Anal. 10: 178-82.

5. Choi HY, Jhun EJ, Lim BO, Chng IM, Kyung SH and Park DK 2000. Application of flow injection- chemilumineacence to the study of radical scavenging activity in plants. Phytother. Res. 14: 250-253.

6. Chu YH, Chang CL and Hsu HF 2000. Flavonoid content of several vegetables and their antioxidant activity. J. Sci. Food and Agri. 80: 561-566.

7. Cotelle N, Bernier JL, Catteau JP, Pommery J, Wallet JC and Gaydou EM 1996. Antioxidant properties of hydroxyl flavones. Free Radiat. Biol. Med. 20: 35-43.

8. Desmarchelier C, Repetto M, Coussio J, Liesuy S and Ciccia G 1997. Antioxidant and prooxidant activities in aqueous extracts of Argentine plants. Int. J. Pharmacog. 35: 116-120.

9. Farjana N, Saud ZA, Rahman MH and Haque ME 2003. In vitro Antimicrobial Activity of the Compound Isolated from Chloroform Extract of Moringa oleifera Lam. Pakistan J. Biol. Sci. 6(22): 1888-1890.

10. Gutteridgde JMC 1995. Free radicals in disease processes: A complication of cause and consequence. Free Radic. Res. Comm. 19: 141- 158.

11. Hamid K, Sultana S, Urmi KF, Ullah MO, Zulfiker AHM and Hossain MA 2011. In vitro free radical scavenging and brine shrimp lithality bioassay of aqueous extract of ficus racemosa seed. Jordan J. Biol. Sci. 4(1): 51-54

12. Jahan IA, Nahar N, Mosihuzzaman M, Begum MR, Ali L, Khan AK, Makhmur T and Choudhary MI 2009. Hypoglycaemic and antioxidant activities of Ficus racemosa Linn. fruits. Nat. Prod. Res. 23(4): 399-408. 
13. Kahl R and Kappus H 1993. Toxicology of the synthetic antioxidants BHA and BHT in comparison with the natural antioxidant vitamin E. Zeitschrift fur LebensmittelUntersuchung und -Forschung 196(4): 329-338.

14. Lean PL and Mohamed S 1999. Antioxidative and antimycotic effects of turmeric, lemon-grass, betel leaves, clove, black pepper leaves and Garcinia atriviridis on butter cakes. J. Sci. Food and Agri. 79: 1817-1822.

15. McDonald LL, Guy RH and Speirs RD 1970. Preliminary evaluation of new candidate materials as toxicants, repellents, and attractants against stored-product insects. 1. USDA Marketing Res. Report 882. Agricultural Research Service, U.S. Department of Agriculture, Washington, DC.

16. Miean KH and Mohamed S 2001. Flavonoid (myricetin, quercetin,kaempferol, luteolin, and apigenin) content of edible tropical plants. J. Agri. Food Chem. 49: 3106-3112.

17. Miller AL 1996. Antioxidant flavonoids: structure, function and clinical usage. Alt. Med. Rev. 1:103-111.

18. Mohd. Zin Z, Hamid AA and Osman A 2002. Antioxidative activity of extracts from Mengkudu (Morinda citrifolia L) root, fruit and leaf. Food Chem. 78: 227-231.

19. Peng ZF, Strack D, Baumert A, Subramaniam R, Goh NK and Chia TF 2003. Antioxidant flavonoids from leaves of Polygonum hydropiper L. Phytochemistry 62: 219-228.

20. Shahidi F, Janitha PK and Wanasundara PD 1992. Phenolic antioxidants. Crit. Rev. Food Sci. Nut. 32: 67-103.

21. Singleton VL, Orthofer R and Lamuela-Raventos RM 1999. Analysis of total phenols and other oxidation substrates and antioxidants by means of Folin-Ciocalteu reagent. Methods in Enzymology 299: 152-178.

22. Sirisha NN, Sreenivasulu MK, Sangeeta C and Chetty M 2010. Antioxident properties of Ficus species-a review. Int. J. Pharm.Tech. Res. 2(4): 2174-2182.

23. Soares JR, Dinis TCP, Cunha AP and Almeida LM 1997. Antioxidant Activities of some Extracts of Thymus zygis. Free Radical Res. 26: 469-478.

24. Talukder FA and Howse PE 1994. Repellent, toxic, and food protectant effects of pithraj, Aphanamixis polystachya extracts against pulse beetle, Callosobruchus chinensis in storage. J. Chem. Ecol. 20: 899-908.

25. Talukder FA and Howse PE 1995. Evaluation of Aphanamixis polystachya as a source of repellents, antifeedants, toxicants and protectants in storage against Tribolium castaneum (Herbst). $J$. Stored. Prod. Res. 31: 55-61.

26. Tiwari AK 2001. Imbalance in antioxidant defence and human disease: multiple approach of natural antioxidants therapy. Curr. Sci. 81: 1179-1187.

27. Viturro C, Molina A and Schmeda HG 1999. Free radical scavengers from Mutsia friesiana (Asteraceae) and Sanicula graveolens (Apiaceae). Phytother. Res. 13(5): 416-418.

28. Willett WC 2002. Balancing life-style and genomics research for disease prevention. Science 296: 695-698.

29. Yagi A, Uemura $\mathrm{T}$ and Okamura $\mathrm{N}$ 1994. Antioxidative sulphated flavonoids in leaves of Polygonum hydropiper. Phytochemistry 34: 885-887.

Manuscript received on 2 December 2013 and revised on 19 December 2013 